Itinéraires Itinéraires

Littérature, textes, cultures

2020-3 | 2021

Le rap, une poésie de performances

\title{
La politique de la parole dans le rap créole : entre enjeux poétiques et engagement littéraire
}

Politics of Speech in Rap Créole: Between Poetic Issues and Literary

Commitment

Lefranc Joseph et Vinson Bradley Noël

\section{OpenEdition}

\section{Journals}

Édition électronique

URL : https://journals.openedition.org/itineraires/9052

DOI : $10.4000 /$ itineraires.9052

ISSN : 2427-920X

Éditeur

Pléiade

Référence électronique

Lefranc Joseph et Vinson Bradley Noël, « La politique de la parole dans le rap créole : entre enjeux poétiques et engagement littéraire », Itinéraires [En ligne], 2020-3 | 2021, mis en ligne le 10 décembre 2021, consulté le 12 décembre 2021. URL : http://journals.openedition.org/itineraires/9052 ; DOI https://doi.org/10.4000/itineraires.9052

Ce document a été généré automatiquement le 12 décembre 2021.

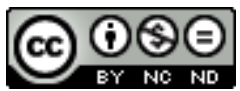

Itinéraires est mis à disposition selon les termes de la licence Creative Commons Attribution - Pas d'Utilisation Commerciale - Pas de Modification 4.0 International. 


\section{La politique de la parole dans le rap créole : entre enjeux poétiques et engagement littéraire}

Politics of Speech in Rap Créole: Between Poetic Issues and Literary

Commitment

Lefranc Joseph et Vinson Bradley Noël

\section{Introduction}

1 Le rap, tel qu'il se pratique en Haïti, est connu sous le nom de rap créole ${ }^{1}$, en référence à sa configuration locale et la langue créole haïtienne dont il est une expression. Les débuts du rap créole coïncident avec l'expansion mondiale de ce genre musical dans les années 1980 où il est sorti de son foyer de naissance dans le Bronx, à New York, pour se répandre à travers le monde. Jusqu'à la fin des années 1980, le rap en Haïti constitue essentiellement une forme de sociabilité créative et de construction de liens sociaux chez des jeunes où oralité et freestyle se pratiquent sous forme de poésie chantée et performative. Ces pratiques, plutôt négligées dans les analyses sur ce genre musical en Haïti, ont servi de base pour fonder et populariser le rap créole au début des années 1990, dans un contexte de libération de la parole qui a succédé à la chute de la dictature en Haïti en 1986.

Dans ses configurations dans le contexte social culturel, politique et économique en Haïti, le rap créole se construit entre politique de la parole, engagement littéraire, poésie discursive et performative. Il y a un aspect fondamental qu'il convient de souligner : dès le début, le rap s'est manifesté comme l'expression poétique et parolière de la culture hip-hop. À ce titre il s'inspire de la tradition orale des cultures africaines (Rose 1994 : 95) et mélange d'une manière originale les instruments traditionnels de la poésie et la musicalité. Dans une relation dynamique entre contexte social, biographies individuelles, expériences sociales multiples, réflexivités et subjectivités, le rap, dans sa version «consciente", vient à se pratiquer comme une mise en scène poétique de la 
parole politique. Les rappeurs utilisent réflexivement les outils poétiques traditionnels pour produire et mettre en scène des narrations (Berry $2018: 32-35)$ et des discours (Colima et Cabezas 2017, Fayolle et Masson-Floch 2002, Roth-Gordon 2009) à résonance politique. Cette relation substantielle entre la poétique du rap et la politique impliquet-elle que l'on doit considérer le rap comme une musique engagée?

Cette question qui renvoie à la nature, aux modalités et implications du rapport entre rap et politique est mal posée dans la mesure où elle se base implicitement sur l'idée fausse que le rap, situé dans une société donnée, peut être caractérisé au moins par un certain degré d'homogénéité sémantique et téléologique. Elle invite en effet à partir de l'idée que le rap est une musique pour ensuite voir si elle est une musique engagée. Ainsi partant de ce présupposé, on est invité à saisir dans une logique d'ensemble le sens et la finalité des prises de position, actions, discours et commentaires du rap sur le monde social. Or, en se basant sur les stratégies de narrations et approches discursives du rap, on peut distinguer de multiples manières de faire du rap, toutes légitimes (Berry 2018 : 32-35, Béthune 2003, Marquet 2013). Cela n'a pourtant pas empêché certains commentateurs et chercheurs de considérer dogmatiquement le rap comme fondamentalement politique, voire révolutionnaire et d'autres d'aller dans le sens contraire et de postuler sous couvert d'une critique paresseuse que le rap est une imposture ou une décadence culturelle et sociale (Deis 2015, Rose 2008).

Qu'en est-il du rap créole ? La relation entre le rap créole et la politique a fait l'objet de nombreux commentaires publics, mais le seul travail de recherche ayant abordé la question de manière systématique semble celui de Jean Evenson Lizaire (2018). L'auteur s'appuie sur des matériaux empiriques substantiels pour soutenir la thèse selon laquelle le rap pratiqué en Haïti, loin d'être une musique engagée, est plutôt une musique de lamentation qui, n'étant pas parvenue à produire un discours politique consistant qui pourrait accompagner un mouvement d'émancipation collective, joue un rôle cathartique pour ses pratiquants.

Le problème avec la thèse de Lizaire (2018) c'est qu'elle essaie de répondre à la question de savoir si le rap créole est une musique engagée en partant d'une normativité de l'engagement. Or, comme nous l'avons mentionné plus haut, cette question se base sur la fausse idée d'une certaine uniformité dans la production des sens et des significations dans ce domaine de production artistique et littéraire. La question, ainsi que la réponse qui s'ensuit, implique une analyse non discriminée tant des différentes catégories narratives du rap que des multiples approches de ces catégories. La question qui s'impose est alors celle qui invite à saisir la place, les manifestations et les modalités de l'engagement politique dans le rap créole.

6 Cet article prend appui sur une série de quatre entretiens semi-dirigés avec un groupe de quatre rappeurs et paroliers, âgés de 35 à 45 ans, vivant dans l'aire métropolitaine de Port-au-Prince ainsi que sur l'analyse du contenu d'un ensemble de chansons et de mises en scène pour proposer une réflexion sur la politique de la parole dans l'univers du rap créole ${ }^{2}$. Les rappeurs ont été sélectionnés pour leur expérience et familiarité avec l'histoire du rap en Haïti. Ces entretiens ont été réalisés à partir d'un guide orientant les échanges sur les points suivants : leur influence, leurs expériences dans le rap créole, leur représentation de l'engagement dans le rap.

7 L'article est divisé en quatre sections. La première saisit les dimensions de la politique de la parole chantée dans le rap créole dans le contexte général de l'avènement de la démocratie en Haïti. La deuxième analyse la politique de la parole dans le rap créole 
comme expression d'une conscience critique, et essaie de comprendre les stratégies de dénonciation et l'exercice de la capacité critique des rappeurs. La troisième examine les mécanismes de la politique et poétique de la parole dans le rap créole. La quatrième, enfin, propose quelques éléments en vue d'appréhender les modalités de l'engagement littéraire dans le rap créole.

\section{6 : Libéralisation de la parole en Haïti et émergence du rap créole}

8 L'année 1986 marque un tournant dans l'histoire de la formation sociale haïtienne. C'est en cette année que Haïti, à l'issue d'une longue période de revendications populaires commencées six ans plus tôt, se libère de la dictature totalitaire des Duvalier dont l'un des piliers était la répression de la parole critique et contradictoire (Hurbon 1979). Cette année constitue ainsi le début de ce qui est connu comme la transition démocratique en Haïti (Midy 1996). Au cœur des mouvements sociaux qui allaient déboucher sur la chute de la dictature se trouvaient des revendications relatives à la liberté d'expression bafouée pendant des décennies (Charles 1994, Inter-American Commission on Human Rights 1963, Trouillot 1986). Ainsi, après le départ du dictateur, ce principe sera codifié dans la Constitution de 1987. Cet épisode dans l'histoire de Haïti est intimement lié à l'émergence du rap créole dans le pays.

9 On observe les premières manifestations du rap en Haïti au cours des années 1980, au (même) moment de la mondialisation de ce style musical (Mitchell 2001). À cette époque, le gouvernement dictatorial de Duvalier utilisait un ensemble de dispositifs pour contrôler les prises de parole et réprimer sévèrement les voix contestataires. Ce contexte social et politique, où certaines productions artistiques, littéraires ou prises de position faisaient fréquemment face à des représailles pouvant mener à l'arrestation, l'emprisonnement et la mort, n'était pas favorable à la construction locale d'un style musical originellement contestataire ${ }^{3}$ (Rose 1994). Il y a deux facteurs qui illustrent les premières manifestations du rap au cours des années 1980. Premièrement, depuis le début de cette décennie, le rap fait partie des éléments qui influencent certaines pratiques musicales en Haïti. Cette influence s'observe notamment dans la manière dont Master Dji, considéré généralement comme le fondateur du rap créole, scande les paroles de la chanson «Vakans» en 1982. Deuxièmement, la plupart des rappeurs qui allaient marquer le rap dans les années 1990 ont tous commencé à le pratiquer depuis les années 1980 (entretien avec Big Boss Blade, Port-au-Prince, 22 mai 2019). Les rappeurs interviewés pour cette étude présentent les manifestations du rap en termes de pratiques sociales de groupe qui entraient dans une logique de sociabilité entre les jeunes. Sur ce versant, le rap était alors défini et vécu comme un domaine où l'activité principale se réduisait à des échanges de lyrics et de flows, des concours de freestyle organisés entre des jeunes en provenance de divers quartiers urbains. Le rappeur Big Boss Blade, qui se dit actuellement retraité et dont le parcours remonte à cette période, nous rapporte que pour lui, ainsi que pour certains de ses camarades et amis, c'était même une stratégie pour aborder certaines matières scolaires comme la physique, les mathématiques ou les sciences sociales (entretien avec Big Boss Blade, Port-au-Prince, 22 mai 2019). Cette stratégie consistait à mettre en lyrics des leçons de sciences sociales ainsi que des démonstrations de formules physiques ou mathématiques, puis les répéter en privé et, ensuite, les scander en face de ses amis. 
10 Ainsi, par-delà l'influence que l'on retrouve notamment chez Master Dji, qui était aussi un DJ, les lieux du rap étaient les groupes d'amis ou les réunions spontanées de jeunes dans les quartiers où les rappeurs, sous l'influence culturelle étasunienne, s'affrontaient au rythme des beatbox humains. C'est là la forme la plus élémentaire de la constitution de l'identité de rappeur en Haïti. Dans ce contexte, l'enjeu c'est la quête de la reconnaissance en tant que rappeur capable et compétent dans la communauté. C'est aussi, en un sens, la lutte pour le pouvoir, la domination dans cette sphère de performance. Ainsi, tout au long des années 1980 jusqu'au tournant des années 1990, le rap en Haïti était pour l'essentiel réduit à des pratiques de sociabilité dans les quartiers. $\mathrm{Au}$ cours de cette période, certains rappeurs, jouissant d'une certaine reconnaissance de leur performance artistique et/ou de faveur d'amis influents, arrivaient à performer devant des publics plus ou moins larges à l'occasion d'animations DJ. Dans ce contexte, sortir une chanson sur cassette ou/et une vidéo était le rêve de tous les rappeurs. Les rappeurs sont les principaux détenteurs de la mémoire de ces pratiques.

11 Au tournant des années 1990, dans le contexte de la libération de la parole dans la société haïtienne, le rap a commencé à se configurer comme un style musical à part entière. C'est au cours de cette période que Master Dji fonde les groupes Haiti Rap'n Raggae, Rap Creole SA et collabore avec de nombreux rappeurs pour produire l'album Match la rèd, en 1994. Dans une chanson du même titre, le fondateur du rap créole et ses collaborateurs décrivent des situations particulières mais typiques des citoyens haïtiens pour illustrer la cherté de la vie, la misère et la corruption, ainsi que leurs conséquences sur la vie sociale. Dès 1990, il a sorti l'album Politik Pa $m$ dont l'un des titres les plus remarquables est «Sispann ». On y retrouve pour la première fois l'usage du sampling tel que systématisé par le rap ${ }^{4}$. À travers cette technique, Master Dji prélève une séquence d'un discours du pape Jean Paul II lors de sa visite en Haïti pour introduire le thème du changement social à la fois comme réalité récente et prospective sociale. Après la séquence «il faut que quelque chose change » qui sert d'intro à la chanson, Master Dji scande : «Chanje, anpil bagay chanje / Depi 7 fevriye Ayiti libere / Jou sa a, tout sa ki te toujou lite/ Ki pandan 29 an pat janm dekouraje ${ }^{5} »$. Cette vision des choses rime avec la vision générale au cours des années qui ont succédé la chute du régime totalitaire duvalierien.

12 À l'époque, le compas, style musical ayant émergé en Haïti dans les années 1950, dominait la scène musicale. Le rap était perçu comme un élément culturel importé, peu raffiné, qui n'y avait pas sa place. Au cours de cette période, les infrastructures de validation et de légitimation de la musique ne s'intéressaient qu'au compas. Que faire du rap ? Devant l'intérêt grandissant pour le rap et la culture hip-hop à la fois du côté des artistes et du public, les producteurs de musique déjà établis ouvrent la porte à ce style musical et s'engagent progressivement dans sa production et promotion. Les structures de production du compas devenaient un espace dominant de validation de la musique rap en Haïti, ce qui allait produire un choc entre la vision des rappeurs et celle des producteurs. Notons que dans les années 1990, l'identité du rap créole comme sphère de performance artistique était déjà plus ou moins affirmée. Ce qui appartient ou non à ce champ est plus ou moins défini par les pratiques et est distingué d'autres pratiques musicales proches, comme le Raggamuffin, populaire en Haïti dans les années 1990. Cette tension allait se manifester dans le processus de sélection des rappeurs et des chansons à produire, distribuer et diffuser. Dans ce processus entrent les questions relatives aux réseaux, les ressources économiques et financières, le contenu parolier. 
Commentant ce processus, le rappeur Blade déclare que « le talent peut être l'élément qui compte le moins» (entretien mené par les auteurs, à Port-au-Prince, le 22 mai 2019). Cette déclaration indique la tension qui a toujours existé entre rappeurs et producteurs, particulièrement quand il s'agit de savoir ce que veut dire avoir du talent dans le domaine du rap. À la fin des années 1990 et tout au long des années 2000, le rap créole allait intensifier sa présence en Haïti à travers la création de nouveaux groupes, studios d'enregistrement, associations de rappeurs, territoires reconfigurés. Tout en s'autonomisant comme espace de production artistique - et donc esthétique - ayant ses dynamiques propres, le rap, tel qu'il se développe en Haïti, reste solidaire des autres formes d'expression artistique qui lui sont complémentaires, particulièrement le graffiti et le Djing.

13 La politique de la parole et de la performance, c'est-à-dire les mécanismes discursifs ou artistiques par lesquels les rappeurs abordent et remettent en cause les modes de traitements politiques des problèmes sociaux, constitue un des piliers de la constitution du rap créole.

\section{Dénonciation et subversion de la parole dans le rap créole social}

14 La politique de la parole dans le rap créole s'articule autour de la dénonciation et de la subversion de la parole, qui constituent un pilier central de ce style musical. Ce pilier peut être analysé comme un critère définitoire du rap créole depuis le début des années 1990 jusqu'à aujourd'hui.

15 La dénonciation renvoie à la tentative parfois singulière, parfois généralisée de pointer du doigt ce qui semble contraire à ce qui devrait être dans la société ; c'est une remise en cause de l'état des choses. Elle consiste en une prise de position contre les maux de la société comme l'injustice, la misère, la violence, la corruption, la dégradation de l'environnement, etc. La dénonciation se présente comme la forme élémentaire de l'engagement politique du rap. Master Dji indique qu'il avait conscience du rôle politique de la dénonciation quand il rappait ces paroles en 1990: "Pale pou denonse mizè ak prejije / Pale pou fé konnen soufrans yo andire ${ }^{6} »$.

Dans le rapport entre prise de parole et fonction sociale du rap en Haïti, la dénonciation s'appuie souvent sur une revendication du droit à la parole critique. Au cours de trois décennies du régime des Duvalier, le dicton «je wè bouch $p e^{7}$ » avait servi à décourager les pratiques discursives dénonciatrices à l'égard du pouvoir dictatorial. À l'époque, une dénonciation publique pouvait entraîner ou bien arrestation, emprisonnement, exil ou bien simplement une exécution sommaire. Même après la chute de la dictature, la culture politique duvaliériste a perduré à un certain degré dans la société haïtienne. Cela permet de comprendre pourquoi l'expression courante "si m pa pale $m$ ap toufe $e^{8}$ " était parfois utilisée, dans les années 1990, par certains rappeurs pour introduire le discours dénonciateur. On la retrouve chez Master Dji en 1990 et aussi chez les Masters of Haiti en 1999. L'expression renvoie aussi à l'idée que les rappeurs, face à une réalité sociale indésirable, croient que la dénonciation est vitale, celle-ci étant présentée sous forme de plaintes, de cris exprimant les souffrances collectives d'un groupe social ou de la société en général. 
17 La parole subversive dans le rap créole constitue une dimension d'approfondissement de la dénonciation. La subversion se distingue de la dénonciation dans le sens qu'elle s'appuie sur cette dernière pour suggérer ou proposer des pistes pour le changement social. Parfois elle s'adresse à la conscience des élites politiques ou économiques, comme on le voit dans la chanson "Ti chans" des Masters of Haiti (1999) ou "Lèt Konsyans » de Blaze One (2019). Dans «Ti chans » [Une chance], les Masters of Haiti, suggérant que la mauvaise gestion de la chose publique, la corruption des dirigeants ainsi que l'intervention intrusive des étrangers sont la cause de la misère du peuple haïtien, demandent « une chance » pour Haïti : «Nou mande yon ti chans pou Ayiti / Nou mande yon ti chans pou sa chanje / Yon ti chans pou lapè ka renye / Yon ti chans pou bon jan van soufle [...]Yon ti chans pou nou jwenn libète / Yon ti chans pou lavichè a bese ${ }^{9}$ ".

Dans «Lèt Konsyans » [Lettre de conscience], le rappeur Blaze One s'adresse directement à la conscience des chefs d'État des vingt dernières années. Il dénonce le fait que les élites politiques et économiques continuent de s'enrichir aux dépens de la majorité de la population qui vit dans une misère de plus en plus exacerbée.

Parfois la subversion prend la forme de prise de position ou d'engagement dans la lutte pour le changement. Elle évite la supplication ou l'appel à la bonne foi des dirigeants, pour aller dans le sens de l'appel à l'action. Comme l'illustrent ces paroles de Barikad Crew, dans ce cas, les rappeurs parlent au nom du peuple: « Nou di non, peyi a pa pou vann / nou pran nan pa konprann / nou gen yon bann otorite se pa nou y ap defann / manje pa janm desann / pa gen travay pou fanm / nou refize met tèt ansanm /lekòl tounen savann ${ }^{10}$ ».

20 La parole subversive a retrouvé une place plus poussée durant les dernières années : le rap est devenu de plus en plus présent dans la société et les commentateurs ainsi que des rappeurs se sont évertués à définir le rap créole et sa fonction dans la société haïtienne. Cette tendance se manifeste dans les pratiques parolières du rap ainsi qu'à travers les discours publics produits par les rappeurs sur leur propre champ artistique. Certains rappeurs se proposent de redéfinir le rap en général et le rap créole comme un champ particulier. Pour ces rappeurs, le rap créole est une critique de la société, par opposition à la vantardise qui domine la chanson de nombreux autres rappeurs comme Izolan ou G Bobby Bon Flow.

21 Cette tendance subversive, dont l'album L'apostat de K-Libr constitue une bonne illustration, s'accompagne d'un métadiscours qui définit le rap par l'engagement artistique dans une perspective proche de la doctrine de l'engagement littéraire (Denis 2005). Cette vision du rap créole exprimée par certains acteurs populaires comme KLibr et d'autres moins populaires comme le groupe Règleman Afè Popilè doit être saisie à la lumière d'une double réflexivité : individuelle et collective. La réflexivité est conçue ici comme l'exercice régulier de la capacité des individus de se penser en relation avec leurs contextes sociaux et vice versa (Archer $2007: 4$ ). C'est à travers l'exercice de la réflexivité que viennent à se constituer, dans le rap, des éléments plus ou moins structurés pouvant fonctionner comme un cadre normatif de production du discours et de la performance dans ce champ qui se manifeste sous la forme d'une politique et d'une poétique du discours et de la performance. 


\section{Politique et poétique du discours et de la performance}

Si l'on examine la discographie du rap à l'échelle mondiale, on se rend compte que les questions pouvant se rapporter à la politique, comme champ où se négocie le processus du gouvernement d'une communauté, constituent seulement une portion de son univers parolier. Force est pourtant de reconnaître que le rap, tant dans ses manifestations globales que localisées, a toujours développé un rapport privilégié à la politique. N'oublions pas que la culture hip-hop a pris naissance dans le Sud du Bronx, dans l'État de New York, à une époque où régnaient la pauvreté et la criminalité. Ce contexte allait inspirer une tendance à la dénonciation et la prise de position radicale dans certaines des premières chansons de rap aux États-Unis d'Amérique. Depuis les années 1980, les commentaires sociaux, critiques à portée politique sont devenus les piliers d'une tendance constitutive du hip-hop en général et du rap en particulier. Celleci est à la base de ce qui a souvent été qualifié de rap conscient, politique, politiquement conscient, militant, contestataire ou engagé (Berry 2018: 32-35; Béthune 2003, 2004 ; Marquet 2013 : 2 ; Rose 1994, 2008 ; Roth-Gordon 2009 : 66).

$\mathrm{Au}$ moment même de la mondialisation du rap dans les années 1980, cette tendance qui représentait une partie significative du hip-hop américain avait occupé le devant de la scène pour dénoncer les inégalités sociales, les disparités raciales mais aussi certaines politiques implémentées par le président Ronald Reagan (1981-1989). C'est au cours de cette période que sont sortis des titres engagés comme "The Message" de Grandmaster Flash \& The Furious Five (1982) ou «Fight the Power » de Public Ennemy (1989).

Dans le contexte haïtien, Lizaire (2014) a bien fait de remarquer les relations entre ville et rap en Haïti. Au moment de la naissance et de l'émergence du rap, Port-au-Prince représentait la communauté urbaine centrale de Haïti au point où on parlait déjà de République de Port-au-Prince pour souligner la centralité de cette ville par rapport au reste du pays. Dans les années de l'affirmation du rap créole en tant champ de production artistique, Haïti était caractérisé, entre autres, par l'instabilité politique chronique, la corruption normalisée, l'exclusion sociale, la violence ordinairement utilisée comme instrument de contrôle politique. Port-au-Prince, où le rap avait déjà commencé à participer à la production de lieux, d'espaces et de territoires, était la scène principale des tumultes politiques. En contact direct avec la réalité qui définit leur quotidien, les rappeurs se trouvent face à une multitude de possibilités narratives et discursives. L'une d'entre elles consiste à narrer, discuter et discourir sur la situation de la société. En conséquence, on retrouve dans les archives du rap créole un univers parolier sur les inégalités, entre autres, la politique ${ }^{11}$, la sexualitée ${ }^{12}$, la violence ${ }^{13}$, la réalité sociale haïtienne des jeunes ${ }^{14}$, la prison ${ }^{15}$, l'histoire ${ }^{16}$. Dans cet univers, les techniques poétiques traditionnelles (rythmes, rimes, versification, figures de style, etc.) sont mises en œuvre pour produire une politique de la parole qui, dans certains cas, met en relation la situation sociale indésirable avec la politique, c'est-à-dire le processus de gouvernance de la communauté, ou, dans d'autres, discute directement de la politique, de ses caractéristiques et implications. Ces deux manifestations de la politique de la parole sont analysées, dans la section suivante, en termes de modalités de l'engagement littéraire dans le rap créole. 


\section{Les modalités de l'engagement littéraire dans le rap créole}

Depuis ses débuts, le rap a su transcender, dans ses multiples pratiques parolières, la dichotomie entre l'oral et l'écrit. En effet, l'élaboration des lyrics, qui constituent l'univers parolier du rap et qui est le lieu où se manifestent des narrations et discours, résulte d'un mélange complexe entre oralité et écriture. Cependant, «en dépit d'un puissant tropisme en direction de l'oral - même s'il s'agit en l'occurrence d'une "oralité seconde"-, la culture hip-hop en général et le rap en particulier demeurent vigoureusement attachés à l'écriture sous toutes ses formes» (Béthune 2011 : 186). Comme le précise Tricia Rose (1994 : 95), même si l'influence de la tradition orale des cultures africaines est profonde dans le rap, il demeure «fondamentalement lettré et profondément technologique ». Dans cette perspective, on peut comprendre que le rap constitue un univers de création artistique qui remet en cause l'opposition traditionnelle entre oral et écrit en les mettant dans « une relation de complémentarité ou, mieux encore, de synergie poétique » (Béthune 2011 : 186). Réfléchissant sur le rap sénégalais, Sophie Moulard-Kouka (2005 : 234) en vient au même constat. Selon elle, le rap attire les jeunes parce qu'il constitue la preuve que «l'on peut écrire comme l'on parle, et même à l'inverse que l'on peut écrire de la poésie (en insistant sur le caractère littéraire du rap) sans la condamner à être figée, en la transposant ensuite dans une oralité vivifiante ». À en croire Béthune (2011 : 186), « la culture hip-hop serait peutêtre la première forme d'expression à entretenir de façon délibérée une tension entre les polarités de l'oral et de l'écrit, et à jouer systématiquement de cette tension dans son processus de créativité poétique ». Dans cette perspective, le rap doit être apprécié comme une innovation littéraire fondée sur un dépassement de l'opposition entre l'oral et l'écrit. C'est une activité à la fois orale et scripturale qui combine de manière originale la tradition orale liée au contexte culturel de sa naissance, les outils technologiques disponibles et les outils poétiques classiques, comme la rime, le rythme, la métrique, les échos phoniques (Barret 2008; Berry 2018 : 2 ; Béthune 2004, 2011 ; Pate 2010 ; Rose 1994 ; Zegnani 2004). Le rap créole n'échappe pas à cette caractérisation générale. Ses tèks ${ }^{17}$ illustrent cette combinaison dynamique et originale entre l'oral et 
l'écrit. Ses pratiques parolières, dans leur double dimension narrative et discursive, sont le résultat d'un travail spécifique sur la langue créole haïtienne sur le plan lexical, sémantique et poétique ${ }^{18}$.

Il va de soi que quand nous parlons d'engagement littéraire dans le rap créole, nous faisons référence à l'appareil parolier du rap politique. En effet, si le travail littéraire est observable dans tous les styles de rap, l'engagement littéraire relève du rap politique. Ainsi, la politique de la parole dans le rap créole se manifeste à partir de deux modalités : la politisation de la parole et la mise en chanson de la parole politique. La politisation de la parole consiste en une double dimension. D'abord, il y a une critique de la gouvernance de la société. Pour politiser la prise de parole, les rappeurs utilisent le rap pour accéder à l'espace public ${ }^{19}$. Ils participent aux débats publics sur des questions variées comme la culture, l'éducation, les inégalités, la violence ou la corruption.

C'est ce que font par exemple les rappeurs de Rap Kreyòl SA (Haïti Rap N Ragga) dans "Match la rèd» (1994). Dans ce titre, les rappeurs font une narration de certains épisodes de la vie des personnages fictifs aux noms haïtiens, Ti Lili, Pòpòl, Bòs Benwa, Manmzèl Roza, Tinès pour décrire la misère qui caractérise la vie ordinaire de personnes réelles. On est là en face d'une mise en scène biographique visant à indiquer les conséquences sociales de la mauvaise gestion des affaires publiques dans la société haïtienne. L'enjeu consiste ici non seulement à insister sur les répercussions biographiques des problèmes sociaux, mais aussi à souligner qu'ils sont les résultats de la gouvernance défaillante. On retrouve cette même stratégie narrative et discursive dans les titres « Pòtoprens » de B.I.C (2010) ou « Vi geto ak lelit» de Blaze One (2017) ${ }^{20}$.

La mise en chanson de la parole politique est plus directe dans la mesure où elle reprend des questions déjà suffisamment politisées. Prenons l'exemple de « $\mathrm{M}$ anvi ri » de K-Libr (2015) et «Lèt Konsyans » de Blaze One (2019). Les deux titres proposent une critique systématique de la politique et des pratiques de gouvernance en Haïti. Dans " M anvi ri », K-libr s'étonne face à la situation sociopolitique incroyable de la société haïtienne au point d'«avoir envie de rire de nous [la société] et de lui-même ». En scandant dans le premier couplet «sa $k$ difisil pou bourik se pase nan yon je zegwi / Men li fasil pou l met vès ak kòl pou l dirije Ayitit ", il critique la situation de corruption et de clientélisme qui rend tellement facile à n'importe qui d'accéder à des postes de responsabilité. Ensuite, il joue poétiquement avec des noms de partis politiques et des noms de candidats ou de responsables politiques pour indiquer que leurs pratiques sont à la base de la misère que "nous " vivons. On retrouve tout cela dans les six premiers vers du deuxième couplet: "Apre maswife nou fin monte ak makout / Nou tonbe nan yon Lavalas ki bwote tout lespwa nou ale/ Nou kite Manman pou kont li n al pran plezi tèt kale / Nou bwè kòb tchup kanaval kounya a tanbou lou / Nan ka sa a 5 plim pat sifi alevwa pou yon K / Nou fè Fusion Mo pa w Mopa m men sa mete n nan plis $\mathrm{ka}^{22}$ ». Utilisant une forme d'antanaclase elliptique, il joue avec le double sens des mots makout, Lavalas, Lespwa, Manman, tèt kale, K-Plim, Fusion, Mopam $^{23}$ et peint un tableau qui illustre les entrelacements entre les problèmes sociaux et le politique.

32 Sur un ton différent, Blaze one, dans sa "Lèt Konsyans ", va dans le même sens. Dans l'introduction même de la lettre, le rappeur en présente méthodiquement le contenu: « Nou rezime tout sa $k$ eksplike povrete / Tout sa $k$ vle di san fyète peyi $n$ se kote pòvrete / Nou wè depi se plen pòch son chwa fasil a vize / Lage geto nan zizani kot espwa l majinalize ${ }^{24}$ ". Comme K-libr, il critique la politique de "plen pòch» (l'avarice des dirigeants 
politiques) en relation avec la pauvreté et la violence. Ici encore, on est en face d'une mise en chanson de la parole politique.

Même si elles sont le résultat d'une forme d'exercice de la réflexivité des rappeurs qui s'expriment en tant que gouvernés et au nom de leurs compatriotes, ces deux modalités sont soumises aux mêmes contraintes du discours politique. Les paroles de « Match la rèd ", " Fòn $\mathrm{n}$ pale ", " $\mathrm{M}$ anvi ri ", «Lèt Konsyans ", par exemple, sont censées exprimer, décrire, dénoncer et critiquer une réalité inacceptable. Comme c'est le cas pour le discours politique (Le Bart $2010: 77$ ), on voit que les gouvernés ont tendance à se reconnaître dans ce qui est dit en leur nom.

\section{Conclusion}

Cet article constitue une tentative de détecter la présence, les caractéristiques et les modalités de la politique de la parole dans le rap créole au regard de ses enjeux poétiques et implications en termes d'engagement littéraire. Nous avons tenté de montrer que la politisation de la parole a toujours constitué une dimension fondamentale dans de nombreuses manifestations de la poétique du rap créole.

La question abordée ici est très complexe et requiert une analyse plus ample qui tienne compte des questions concernant les déterminants ainsi que les implications de la politique de la parole et de la performance poétiques dans le rap créole. Il peut être exagéré de considérer le rap créole comme une musique purement ou strictement engagée. Il convient plutôt, dans une démarche analytique, de comprendre que la politisation de la parole poétique et la mise en poésie de parole politique ont toujours été une dimension constitutive du rap créole. Autrement dit, si le rap créole n'est pas une musique engagée, l'engagement politique constitue un élément fondamental de la poétique du rap. La question de connaitre les impacts ou les implications sociales et politiques de cette politisation de la parole est importante et déborde le cadre de cet article. Cela ouvre cependant la voie pour comprendre les modalités de l'engagement littéraire dans le rap créole.

Par ailleurs, cette démarche permet aussi de saisir la multiplicité des poétiques de la parole dans le rap créole et la tension qui pourrait exister entre elles. En effet, chez un même rappeur, un même groupe et parfois dans un même album, on peut trouver la coexistence contradictoire de la tendance politique et de la tendance ludique ou vantarde. L'analyse de cette tension, que nous n'avons pu prendre en compte dans cet article, pourrait aider à comprendre les multiples fonctions et usages de la poétique du rap créole.

\section{BIBLIOGRAPHIE}

Archer, Margaret S., 2007, Making our Way Through the World: Human Reflexivity and Social Mobility, Cambridge, Cambridge University Press. 
Barret, Julien, 2008, Le rap ou l'artisanat de la rime : stylistique de l'egotrip, Paris, L'Harmattan. Barthéléemy, Gérard, 2000, Créoles-Bossales. Conflit en Haïti, Petit-Bourg, Ibis Rouge. Berry, Michael, 2018, Listening to Rap, New York, Londres, Routledge. Béthune, Christian, 2003, Le Rap : une esthétique hors la loi, Paris, Autrement. Béthune, Christian, 2004, Pour une esthétique du rap, Paris, Klincksieck. Béthune, Christian, 2011, « Sur les traces du rap », Poétique, vol. 166, nº 2, p. 185-201. Casimir, Jean, 2001, La culture opprimée, Delmas, Imprimerie Lakay. Charles, Etzer, 1994, Le pouvoir politique en Haïti de 1957 à nos jours, Paris, Khartala. Colima, Leslie et Cabezas, Diego, 2017, « Analysis of Social Rap as a Political Discourse of Resistance ", Bakhtiniana, vol. 12, $\mathrm{n}^{\circ}$ 2, p. 25-44.

Deis, Christopher, 2015, « Hip-Hop and Politics », dans J.A. Williams (dir.), The Cambridge Companion to Hip-Hop, Cambridge, Cambridge University Press, p. 192-205.

Denis, Benoît, 2005, « Engagement littéraire et moral de la littérature », dans E. Bouju (dir.), L'engagement littéraire, Rennes, Presses universitaires de Rennes.

Dirksen, Rebecca, 2019, After the Dance, the Drums Are Heavy. Carnival, Politics, and Musical Engagement in Haiti, Oxford, Oxford University Press.

Fayolle, Vincent et Masson-Floch, Adeline, 2002, « Rap et politique », Mots, n 70, p. 79-99.

Hurbon, Laënnec, 1979, Culture et dictature en Haïti. L'imaginaire sous contrôle, Paris, L'Harmattan. Inter-American Commission on Human Rights, 1963, Report on the Situation of Human Rights in Haiti, [En ligne], http://www.cidh.oas.org/countryrep/Haiti79eng/toc.htm.

Le Bart, Christian, 2010, « Parler en politique », Mots, n 94, p. 77-84, [En ligne], https://doi.org/ 10.4000/mots.19867.

Lizaire, Jean Evenson, 2014, « La résonance biographique du rap : entre sens commun et communauté de sens ", Le Sujet dans la Cité, vol. 2, n 5, p. 201-213.

Lizaire, Jean Evenson, 2018, La pratique du rap en Haïti : un lieu d'autoformation et de subjectivation, Thèse de doctorat, Université Paris 13.

Marquet, Mathieu, 2013, « Politisation de la parole : du rap ludique au rap engagé », Variations, $\mathrm{n}^{\circ}$ 18, [En ligne], https://doi.org/10.4000/variations.645.

Midy, Franklin, 1996, « La transition vers la démocratie : lever les obstacles ou poser les fondements ? ", dans L. Hurbon (dir.), Les transitions démocratiques. Actes du colloque international de Port-au-Prince, Haïti, Paris, Les Éditions Syros, p. 181-202.

Mitchell, Tony (dir.), 2001, Global noise: Rap and hip-hop outside the USA, Middletown, CT, Wesleyan University Press.

Moulard-Kouka, Sophie, 2005, « Le rap sénégalais : un mode d'expression inédit entre oral et écrit », Matatu. Journal for African Culture and Society, n 31-32, p. 233-246.

Pate, Alexs, 2010, In the Heart of the Beat: The Poetry of Rap, Lanham, The Scarecrow Press.

Rose, Tricia, 1994, Black Noise: Rap Music and Black Culture in Contemporary America, Middletown CT, Wesleyan University Press. 
Rose, Tricia, 2008, The Hip Hop Wars. What We Talk About When We Talk About Hip Hop-and Why It Matters, New York, Basic Civitas.

Roth-Gordon, Jennifer, 2009, « Conversational Sampling, Race Trafficking, and the Invocation of the Gueto in Brazilian Hip Hop », dans H. S. Alim, A. Ibrahim et A. Pennycook (dir.), Global Linguistic Flows Hip Hop Cultures, Youth Identities, and The Politics of Language, New York, Londres, Routledge, p. 63-78.

Trouillot, Michel-Rolph, 1986, Les racines historiques de l'État duvaliérien, Port-au-Prince, Henri Deschamps.

Zegnani, Sami, 2004, «Le rap comme activité scripturale : l'émergence d'un groupe illégitime de lettres ", Langage et société, vol. 110, nº 4, p. 65-84, [En ligne], https://doi.org/10.3917/ls.110.0065.

\section{NOTES}

1. Certains avancent que le qualificatif "créole» dans rap créole renvoie à la dichotomie créoles/bossales théorisée notamment par Gérard Barthélémy (2000) Jean Casimir (2001) et préfèrent faire du rap bossale (Lizaire 2018: 52-56). Telle est la position du groupe Règleman Afè Popilè, fondé dans les années 2010 par des étudiants de l'Université d'État d'Haïti familiers au cadre théorique de Barthélémy (2000) et de Casimir (2001). Cela a poussé Jean Evenson Lizaire (2018: 56) à parler plutôt de rap haïtien, terme qui lui «semble beaucoup moins ambigu et plus opérationnel». En réalité, le rap créole, traduction française de rap kreyòl, en créole haïtien, semble renvoyer plutôt à la nature spécifiquement locale et artisanale du rap produit en Haïti. C'est aussi en ce sens qu'en Haïti, particulièrement dans les milieux défavorisés, on parle de dlo kreyòl (eau créole) pour identifier de l'eau potable mise en sachet artisanalement et vendue au détail, ou de zam kreyòl (arme créole) en référence à une arme à feu construite de façon artisanale.

2. Les quatre personnes interviewées sont: Big Boss Blade, Ali Replikan, Sejan Tòkèt et Raslo. Yvens Codio de son nom d'artiste, Big Boss Blade, est né en 1974 à Port-auPrince. Il a fait ses débuts dans le rap créole au début des années 90 au sein du groupe True Negaz, ensuite il a fait partie de différents groupes tels que : First Clan, Grave Clan, 2-Pat-Cha et de Moun Fou Lokal. Il a collaboré avec différents artistes et groupes à succès dont Masters of Haiti et Shakka Dreams. Alexandre Jacques Eli (Ali Replikan) est né en 1977 à Port-au-Prince. Son premier groupe est DFS fondé en 1992, ensuite il a fait partie de Double Power Squad puis de Boule Mic. Il a participé à différents albums avec des groupes connus comme Masters of Haiti. Jerry Reginal Chery (Sejan Tòkèt) a un parcours dans le rap créole qui tourne autour des groupes Vol 404, Getho Ipap et LSPS (Lame San Pran Souf). Phillipe Monteleau (Raslo) est né en 1976. Il a été très actif dans le rap en Haïti. Il a collaboré avec le groupe Five Staff et Getho Ipap dans les années 1990. Il a formé le groupe LSPS (Lame San Pran Souf) avec des amis. Avec ce groupe, il a produit un EP de 4 titres qui a eu un certain succès dans la communauté du rap en Haïti. Les entretiens ont été réalisés entre mai et août 2019.

3. La contestation est un élément fondamental dans la définition du rap. En effet, les chansons fondatrices les plus popularisées de ce genre musical traitaient largement des questions relatives à la pauvreté, le racisme, l'exclusion et la violence vécus par les minorités noires dans le Bronx (New York). Ce serait cependant une erreur 
réductionniste que de croire que le rap se limite à la contestation (Berry 2018:3235, Béthune 2003, Marquet 2013).

4. Le sampling est une technique qui consiste à prélever numériquement une séquence sonore mélodique ou discursive, un fond rythmique, une ligne instrumentale, etc. sur un enregistrement préalable et à l'utiliser dans une chanson pour produire de nouveaux effets sonores et sémantiques.

5. «Changer, beaucoup de choses ont changé / Depuis la libération d'Haïti le 7 février / Ce jour, tous ceux qui ont toujours lutté /qui, pendant 29 ans [de la dictature] n'ont pas découragé ».

6. «Parler pour dénoncer la misère et la discrimination / parler pour faire connaitre les souffrances qu'ils [les Haïtiens] endurent ».

7. «Observer sans parler».

8. «Je ne parle pas, je serai étouffé».

9. « Nous demandons une chance pour Haïti / Nous demandons une chance pour que ça change / Une chance pour que la paix règne / Une chance pour que vous vienne du bon vent [...] Une chance pour que nous trouvions la liberté / Une chance pour que baisse le coût de la vie ».

10. «Nous disons non, le pays n'est pas à vendre / On nous a bernés / Les autorités ne défendent pas nos intérêts / La vie est chère / les femmes sont au chômage / Nous refusons de nous unir / L'école, c'est la galère ».

11. Par exemple « Yon ti chans » (Masters of Haiti, 1999); M anvi ri (K-Libr, 2015).

12. «Banm afè $m$ » (Barikad Crew, 2007).

13. «Gran Dosye » (Blaze One, 2016).

14. «Pòprens Mafia » (Masters of Haiti, 1999).

15. «Prizon federal » (Blaze One, 2012).

16. « Rap prezidans » (Doc Filah feat. Bic, 2013), « Istwa Dayiti » (Blaze One, 2016).

17. Le terme tèks, en créole haïtien, est souvent utilisé par les rappeurs haïtiens pour se référer au contenu parolier d'une chanson.

18. Cet aspect littéraire dans le rap créole est parfois revendiqué par certains rappeurs comme BIC et K-Libr.

19. L'accès à l'espace public à travers le rap est observable depuis les années 1990. Par exemple, grâce à certains titres contestataires, les Masters of Haiti sont devenus de véritables protagonistes des débats publics.

20. Dans « Pòtoprens », B.I.C (2010) rappe « Èske jou koulye / Ti fifi ap kouche pou plat manje?» [Est-ce que jusqu'à présent Ti Fifi se prostitue pour de la nourriture]. Similairement, dans «Vi geto ak lelit » Blaze One scande : "Se nan geto pouw wè1 lè Ti Mari antre sil pa pot bon nouvèl Bòs Djo pral tiye 1 a kout pye » [C'est dans la banlieue que vous verrez que quand Ti Marie rentre à la maison, si elle ne ramène pas de bonnes nouvelles Bòs Djo lui donnera des coups de pied »].

21. «Ce qui est difficile à l'âne c'est de pouvoir passer dans le chas d'une aiguille/ Alors qu'il lui est facile d'enfiler son costard pour diriger Haïti. »

22. «Après avoir vécu des moments difficiles/ L'avalanche [en référence au parti politique du nom de Lavalas/L'avalanche] a emporté tous nos espoirs [en référence au parti politique Espwa/Espoir] / Nous avons délaissé la mère pour aller festoyer tèt kale 
[ici la mère symbolise la candidate de 2010 à la présidence Mirlande Manigat, caractérisée par ses compétences intellectuelles et politiques, en face du candidat Michel Joseph Martelly qui a remporté les élections dont le slogan est Tèt Kale et qui se caractérise par son immaturité politique] / Nous avons beaucoup festoyé durant le carnaval maintenant c'est le temps des vaches maigres/ Que faire d'un $\mathrm{K}$ quand 5 plumes n'aurait suffi [en référence à un récent premier ministre surnommé K-Plim] / Nous avons fait Fusion, ton mot, mon mot mais ça nous enfonce beaucoup plus [Fusion et Mopam/Mopod sont des partis politiques en Haïti]. »

23. Tous ces noms créoles sont à la fois des noms propres et communs. Makout veut dire sac, besace, mais renvoie aussi aux Tontons Macoutes qui étaient une force paramilitaire brutale créée en 1959 par le dictateur François Duvalier. Lavalas veut dire avalanche, c'est aussi le nom d'un parti politique très influent (Fanmi Lavalas) fondé en 1996 par l'ancien président Jean Bertrand Aristide. Lespwa signifie espoir. Dans le texte le terme fait aussi référence au parti politique fondée en 2005 par l'ancien président d'Haïti, René Garcia Préval. Manman, qui veut dire maman, prend aussi un sens politique dans ce fragment. Au cours des élections générales haïtiennes de 2010-2011, l'expression «Ban m manman $m$ » (Donnez-moi ma mère) attribuée à la candidate à la présidence Mirlande Manigat, s'opposait au refrain insouciant « Tèt Kale » (tête rasée) Michel Joseph Martelly (Voir Dirksen 2019 : 176). K-Plim est un sobriquet de Evans Paul, politicien haïtien de longue carrière et ancien premier ministre sous la présidence de Martelly. Le terme Fusion est utilisé dans le sens propre et en même temps il renvoie au parti politique Fusion des Sociaux-Démocrates Haïtiens, fondée en 2005. MOPAM sonne comme mo pa m c'est-à-dire mon mot. C'est aussi le sigle du parti politique Mouvement Progressiste pour l'Avancement des Masses.

24. Nous avons résumé tout ce qui explique la pauvreté / Tous ce qui se rapporte au déshonneur du pays habite les pauvres / Nous comprenons dès qu'il s'agit de se remplir les poches le choix est facile / Créer la zizanie dans le ghetto là où l'espoir est marginalisé.

\section{RÉSUMÉS}

Cet article examine les manifestations de la politique de la parole en tant qu'élément constitutif du rap pratiqué en Haïti. S'appuyant sur les propos de rappeurs ainsi que sur l'analyse de certaines pratiques narratives et discursives observées dans le rap créole depuis des années 1990, l'analyse permet de montrer que la politique de la parole a toujours été un élément constitutif de ce champ de création artistique et littéraire. Nous examinons le rap créole au regard de l'idée de l'engagement politique et avançons que s'il n'est pas possible d'affirmer que le rap créole est une musique engagée, l'engagement politique constitue un élément fondamental de sa poétique. Enfin, nous analysons la politisation de la parole et la mise en musique de la parole politique comme deux modalités de l'engagement littéraire dans le rap créole.

This article examines the manifestations of the politics of speech as a constitutive element of Haitian rap. Based on interviews with rappers as well as the analysis of narrative and discursive 
practices observed in Haitian Rap Créole since the 1990's, the article shows that the politics of speech has always been a constitutive element of this field of artistic and literary creation. We examine Haitian Rap Créole in terms of the idea of political engagement. We argue that although it is not possible to assert that Rap Créole is entirely political, political engagement is central to its poetics. Finally, we analyze the politicization of speech and the setting to music of political rhetoric as the two modalities of literary engagement in Rap Créole.

\section{INDEX}

Keywords : rap créole, rapper, politics, poetics, literary commitment, political commitment, Haiti, performance art, urban art, Haitian Creole

Mots-clés : rap créole, rappeur, politique, poétique, engagement littéraire, engagement politique, Haïti, art de performance, art urbain, créole

\section{AUTEURS}

\section{LEFRANC JOSEPH}

Département de sociologie, Faculté des Sciences Humaines, Université d'État de Haïti et Centre haïtien de recherche en sciences sociales (Charesso)

\section{VINSON BRADLEY NOËL}

Centre de Recherche d'Intervention en Suivi Communautaire (CRISC) 